\title{
Research on the Design Method of the Starting Process of Main Fuel Control system for Aviation Auxiliary Power Unit
}

\author{
Yanan Guo ${ }^{1, a}$, Ding Fan ${ }^{1}$, Kai Peng ${ }^{1}$, Fang Tian ${ }^{1}$ \\ ${ }^{1}$ School of Power and Energy, Northwestern Polytechnical University, Xi'an Shaanxi, China
}

\begin{abstract}
This paper analyzed the main fuel control system of the auxiliary power unit (APU), explored the starting process of the main fuel flow control system control law and control algorithm. Focus on analyzing the interpolation table that is involved in the controller of the starting process, restoring its physical meaning. The design method of the main fuel flow controller for the starting process of the APU is summarized. It's a valuable reference for the future design of the control system of both aero-engine and auxiliary power unit.
\end{abstract}

\section{Introduction}

Auxiliary power unit (APU) is a set of independent small gas turbine power plant which is installed on the aircraft. It is not depend on any energy from outside the machine, and mainly used for output electric power, shaft power and pressurized gas source, in order to meet modern aircraft the engine start, the air emergency, the cabin air conditioner and other auxiliary energy supply requirements. APU is an important and indispensable part of today's aircraft systems. Based on a certain type of single rotor full authority digital electronic control system of auxiliary power unit, this type of APU's structure and working principle are analyzed; the starting process of main fuel flow control law and control algorithm are studied; explored some parameters and interpolation table meaning, completed the main fuel control system design method of the APU starting process analysis and research work.

\section{APU structure and working principle}

The APU belongs to single rotor structure, mainly including two basic parts which is APU core engine and transmission gear box. For the structure, the core engine is a single-stage centrifugal plus vane diffuser compressor, reflux annular combustor and a single-stage radial turbine. The compressor and turbine are connected coaxial, while the exhaust pipe which is of diffusion structure has a muffler to reduce the noise of the highspeed gas. Transmission gearbox will reduce the speed of high-speed turbine, and connect the starter, fuel systems, oil system, generator and cooling fan together. APU operating mode includes start mode, available mode, main engine start mode, loop control mode and stop mode.

\section{The main fuel control law of starting process}

\subsection{The Simulink model of the main fuel flow control system}

We established the main fuel control system simulation model of the APU in MATLAB/Simulink, as shown in Figure 1.

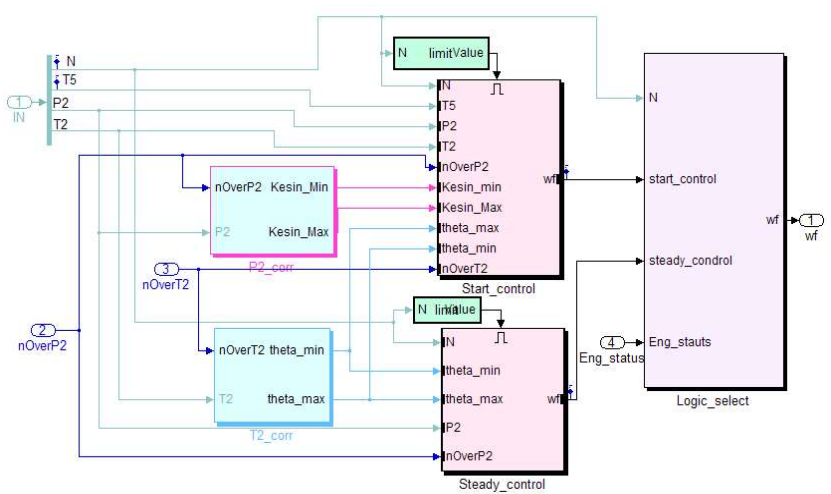

Figure 1. The Simulink model of the main fuel flow control system

As the figure shows, the fuel flow control system simulation model is divided into four modules, namely correction calculation module, calculation module, the

\footnotetext{
a Yanan Guo: guoyanan0002@163.com
} 
starting process steady state process calculation module and control logic and control mode selection module.

\subsection{The control law of the starting process}

The type of the APU in starting process is controlled by exhaust temperature and the current speed $\mathrm{d}^{\text {jusf }}$ as shown in Figure.2. The control law is: ${ }_{f}=f\left(T_{5}, n\right)$, and the exhaust gas temperature ${ }^{T_{5}}$ is the controlled variable, the fuel flow ${ }_{f}$ is control variable. Controller first according to the size of the current APU rotor rotational speed calculates the corresponding expectation value of exhaust temperature, and subtracts the expected exhaust gas temperature and the current exhaust temperature. Then the controller according to the current APU rotating speed and the fuel electric current which compute from the inlet condition calculate the upper and lower limits, then calculated according to the current APU rotating speed and inlet condition fuel electric current of the upper and lower limits, and then through the variable parameter PI algorithm to calculate the fuel torque motor current value, and then get the current time needed by the APU fuel flow.

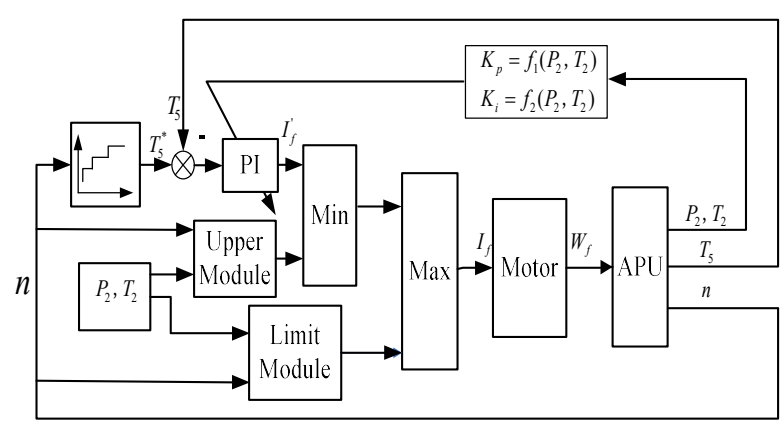

Figure 2. The main fuel flow control law of starting process

\section{The analysis on the starting process control algorithm and the design method}

\subsection{PI controller algorithm}

The starting process fuel flow calculation used in the incremental change control mode, proportional coefficient and integral coefficient are changed with the APU compressor inlet air pressure and temperature, it is shown in formula 1. $k_{p}, k_{i}$ are constant. $T_{2}$ corr is the compressor inlet temperature correction coefficient, $P_{2}$ corr is the compressor inlet pressure correction coefficient. $f_{1}$ and $f_{2}$ is respectively express interpolation table 1 and interpolation table 2.

$$
\left\{\begin{array}{l}
K_{P}=\frac{f_{1}\left(P_{2} \_ \text {corr }\right)}{T_{2} \text { corr }} \times k_{p} \\
K_{\mathrm{I}}=\frac{f_{2}\left(P_{2} \text { corr }\right)}{T_{2} \text { corr }} \times k_{i}
\end{array}\right.
$$

According to the data interpolation table 1 and interpolation table 2 features its fits the characteristics of exponential function, we find that the exponential function is adopted for fitting interpolation table. Then we use the least square method to optimize the fitting parameters.The fitting formula and fitting function curve are shown in Figure. $3^{\sim} 4$. Then we sum up the expression of proportion and integral coefficient, it is shown in formula 2. $P_{s t d}$ is the standard atmospheric pressure, $P_{s t d}=14.7 \mathrm{psia}$. When $P_{2}>P_{\text {std }}, P_{2}=P_{\text {std }}$. $T_{\text {std _ corr }}$ is the correction of standard atmospheric pressure for atmospheric temperature, $T_{\text {std }}$ corr $=288.15 \mathrm{~K} . c_{3}$ and $c_{4}$ are fitting parameters.

$$
\left\{\begin{array}{l}
K_{p}=k_{p}\left(\frac{P_{2}}{P_{s t d}}\right)^{c 3}\left(\frac{T_{2 \_} \text {corr }}{T_{s t d} \text { corr }}\right)^{-1} \\
K_{i}=k_{i}\left(\frac{P_{2}}{P_{s t d}}\right)^{c 4}\left(\frac{T_{2} \text { corr }}{T_{\text {std }} \text { corr }}\right)^{-1}
\end{array}\right.
$$

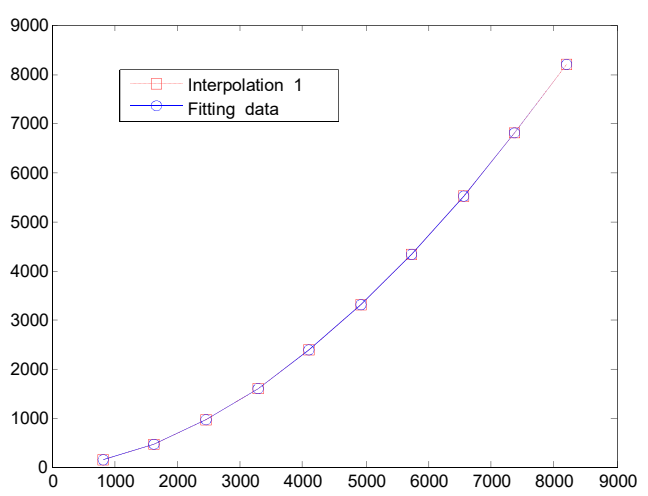

Figure 3. The fitting curve of interpolation table 1

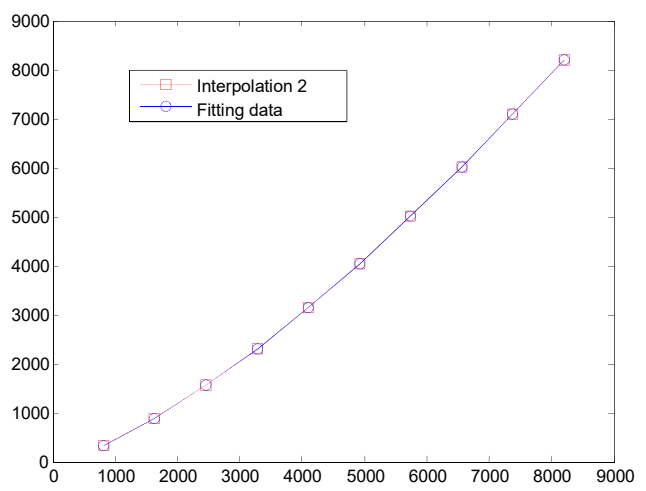

Figure 4. The fitting curve of interpolation table 2 


\subsection{The design method of the proportion coefficient and integral coefficient of the starting process}

To explore the design method of proportional integral coefficient, we choose the formula 3 as the objective function. Firstly, we use the genetic algorithm to adjust the proportion coefficient and integral coefficient in ground standard conditions, scale integral coefficient was adjusted using genetic algorithm. $w_{1}, w_{2}, w_{3}$ are the different weights. $e$ is the deviation between the expected and actual turbine rear temperature. $t_{u p}$ is the time for APU to start up to $98 \%$ rotational speed. $g$ is the punishment term, and the specific forms are as shown in formula 4.

$$
\begin{gathered}
J=\int_{0}^{t}\left(\omega_{1}|e|+\omega_{2} u^{2}(t)\right) d t+\omega_{3} \cdot\left(25-t_{u p}\right)+g \\
g= \begin{cases}K\left(n_{s}-105\right) & , n_{s} \geq 105 \% \\
K\left(97-n_{s}\right) & , n_{s} \leq 97 \% \\
K\left(T_{5}-105\right) & , T_{5} \geq 690^{\circ} \mathrm{C}\end{cases}
\end{gathered}
$$

Referring to the process of optimizing the proportion constant and integral constant under the ground standard condition, the proportional coefficient and integral coefficient are set by genetic algorithm and objective function under the different conditions. The proportion coefficient and integral coefficient of these operating points are obtained as shown in Figures $5 \sim 6$.

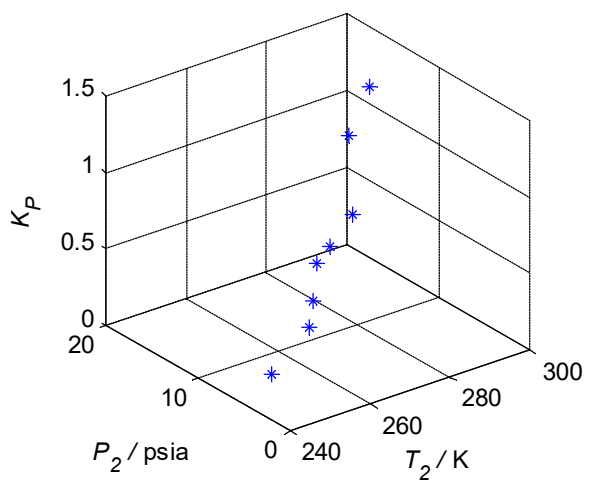

Figure 5. The proportion coefficients of different operating points are optimized

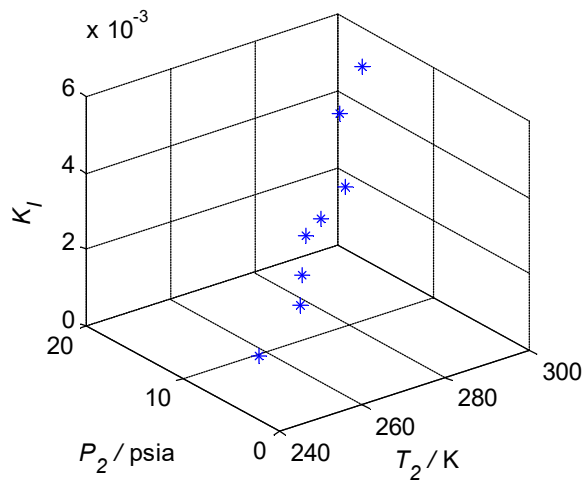

Figure 6. The integral coefficients of different operating points are optimized

By analyzing Figure $5 \sim 6$, it can be seen that the variation trend of optimized proportion coefficient and integral coefficient are similar, and it conforms to the variation form of exponential function. By comparison with formula 2 it can be seen that the fitting result is very close to the index of $\left(P_{2} / P_{s t d}\right)$ used in the APU control system, and the value of $\left(T_{2}\right.$ corr $/ T_{\text {std }}$ corr $)$ is 1. Therefore, it is speculated that in the design of the APU control system, the exponential value of $\left(T_{2}\right.$ ccorr $/ T_{\text {std }}$ corr $)$ is fixed to -1 , and only the exponent of $\left(P_{2} / P_{s t d}\right)$ is optimized.

Through the above analysis, the design process of the starting process proportion coefficient and integral coefficient can be summarized as follows. At different operating points within APU's entire envelope designed the proportion coefficient and integral coefficient respectively, which satisfied the performance requirements of APU. Then use functions to fit these coefficients. However, it is difficult to fit the proportion coefficient and integral coefficient of all working points accurately by using the function, so the fitting precision of some points is usually sacrificed. In order to facilitate the design, the proportion coefficient and integral coefficient of the design point in the standard condition are taken as constants, the exponential value of $\left(T_{2}\right.$ corr $/ T_{\text {std }}$ corr $)$ is fixed to -1 , only the exponent of $\left(P_{2} / P_{s t d}\right)$ is optimized. In addition, considering the limitations of hardware, the optimized results are arranged in the form of interpolation table to improve the calculation speed.

\section{Conclusions}

The full authority digital electronic control system of a certain single rotor auxiliary power unit is taken as a research object. Combined with the working principle of APU in the environment of Simulink, a simulation model of the main fuel control system was built. The control law and the control algorithm of the main fuel 
flow were derived by reverse analysis combined with the working principle and the recorded data of working process of the APU. The variable parameter PI algorithm in the main fuel flow control algorithm is analyzed and designed. Through the exploration of some parameters and interpolation tables, the design method of starting process of the APU main fuel controller is summarized. Analysis results can provide valuable reference for the future design of the control system of engine and auxiliary power unit.

\section{References}

1. Li Dongjie. Application Status and Development Trend of Auxiliary Power Unit: Aviation Science and Technology, 2012.

2. Fan Siqi. Aero engine control [M]. Xi'an: Northwestern Polytechnical University Press, 2008.
3. Yao Hua. Full authority digital engine control system [M]. Beijing: Aviation Industry Press, 2013.

4. Fangyuan Lou. Experimental Study of Flow Field in Rectangular Sectioned 90 Degree Bend of an APU Style Inlet (AIAA 2015-3701), 2015.

5. CHEN L, HUO $Y L$. On solution to nonlinear equation group by means of improved genetic algorithm[J]. Journal of Southwest China Normal University (Natural Science Edition), 2015.

6. JOSHI G, KRISHNA M B. Solving system of nonlinear equations using genetic algorithm[C], International Conference on Advances in Computing, Communications and Informatics. Pis-caraway, NJ: IEEE Press, 2014. 